\title{
Attention fluctuations impact ongoing maintenance of information in working memory
}

Nicole Hakim ${ }^{1,2 *}$, Megan T. deBettencourt ${ }^{1,2}$, Edward Awh ${ }^{1,2,3}, \&$ Edward K. Vogel ${ }^{1,2,3}$

${ }^{1}$ Department of Psychology, University of Chicago, Chicago, Il.

${ }^{2}$ Institute for Mind and Biology, University of Chicago, Chicago, Il.

${ }^{3}$ Grossman Institute for Neuroscience, Quantitative Biology, and Human Behavior, University of Chicago, Chicago, Il.

*Corresponding author:

Nicole Hakim

940 E $57^{\text {th }}$ St.

Chicago, IL 60637

nhakim@uchicago.edu, 773-795-4784

Acknowledgments Research was supported by NIH grant ROIMH087214 and Office of Naval Research grant N00014-12-1-0972 and NIH fellowship F32MH115597. We thank K.C.S. Adam for feedback on the study design and K. Oberauer for feedback on the computational model and the manuscript.

Author contributions: N.H. and E.K.V conceived of the study. N.H. and M.T.dB. performed analyses. N.H. collected the data and wrote the initial draft of the manuscript, which all authors read and edited.

Data availability: Datasets for all experiments will become available online on Open Science Framework upon acceptance of the manuscript or reviewer request.

Conflicts of Interest: none

Keywords: short-term memory, retention interval, whole report, computational modeling, sustained attention 


\begin{abstract}
Working memory maintains information in a readily accessible state and has been shown to degrade as the length of the retention interval increases. Previous research has suggested that this decline is attributable to changes in precision as well as sudden loss of item representations. Here, by measuring trial-to-trial variations in performance, we examined an orthogonal distinction between the maximum number of items that an individual can store, and the probability of achieving that maximum. Across two experiments, we replicated the finding that performance declines after long (10 second) retention intervals, as well as past observations that forgetting was due to probabilistic dropping of individual items rather than all-or-none losses of the stored memories. Critically, longer retention intervals did not reduce the maximum amount of information that could be stored in working memory. Instead, lower attentional control accounted for a decreased probability of maintaining the maximum number of items in working memory. Thus, longer retention intervals impact working memory storage via fluctuations in attentional control that lower the probability of achieving a stable maximum storage capacity.
\end{abstract}


Visual working memory facilitates the temporary maintenance of information over time for use in ongoing cognitive processes. The majority of working memory research has examined performance over short ( $\sim 1$ second) retention intervals. However, there is growing evidence that suggests that working memory performance declines after longer ( $\sim 10$ seconds) retention intervals (Donkin, Nosofsky, Gold, \& Shiffrin, 2015; Rademaker, Park, Sack, \& Tong, 2018; Zhang \& Luck, 2009). ${ }^{1}$ These studies have mostly focused on whether this decline in performance is due to changes in precision or due to the sudden loss of information over time. In the present work, however, we will focus on an orthogonal distinction that further characterizes how increasing retention intervals affect performance. Specifically, we will dissociate how attentional control and working memory capacity contribute to working memory performance over long retention intervals.

Previous work has investigated the intricate relationship between attention and working memory performance (Adam, Mance, Fukuda, \& Vogel, 2015; Awh, Vogel, \& Oh, 2006; De Fockert, Rees, Frith, \& Lavie1, 2001; deBettencourt, Keene, Awh, \& Vogel, 2019). This work has provided evidence that trial-by-trial variability in attention contributes to individual differences in working memory performance. One such study deployed a whole report procedure, in which subjects reported the identity of each item from the memory display on every trial (Adam et al., 2015). They found that differences in visual working memory performance both across and within individuals over short retention intervals were well described by a combination of two separate parameters: Kmax, maximum working memory capacity, and $a$, a factor related to attentional control (Adam et al., 2015). Given that these two parameters, Kmax and $a$, explain performance

\footnotetext{
${ }^{1}$ Of note, there are also a handful of studies have found no effect of retention interval length on working memory performance (Lewandowsky \& Oberauer, 2009; Oberauer \& Lewandowsky, 2008).
} 
variability at short retention intervals, in our study, we are interested in whether the decline in performance observed after long retention intervals is due to changes in Kmax, $a$, or some combination of these two parameters.

Another unresolved concern is that we do not know how long retention intervals act on multiple representations because previous research has utilized partial report tasks, in which a single item from the display is randomly chosen to be tested. In particular, there are two possible alternatives for performance declines at long retention intervals: (1) participants could drop all information from working memory (complete drop) or (2) participants could drop individual items (partial drop). One task that is well suited to examine representations of multiple items on a single trial is a discrete whole report working memory task. This task requires participants to click among discrete colors to report the identity of each item in the display (Adam et al., 2015). Performance on discrete whole report tasks can range from getting no items correct, to getting all items correct, or anything in between. Across individuals, average discrete whole report performance is highly correlated with performance on traditional change detection tasks, where a single item is randomly probed (Adam et al., 2015). However, the discrete whole report task has substantial advantages because it measures memory for each item on a multi-item display. This allows us to assess whether changes in performance after long retention intervals are due to completely dropping all information from working memory or due to an increased likelihood of dropping individual items.

In the current study, we had participants perform a discrete whole report task while maintaining information over long (10 s) and short $(1.5 \mathrm{~s})$ retention intervals. To analyze the data, we designed a computational model that analytically solved for attentional control $(a)$ and maximum working memory capacity $(K \max )$, which we based on simulations from a previous paper (Adam et al., 2015). We were interested in (1) characterizing how the distributions in the 
number of items remembered varied across short vs. long retention intervals, and (2) using these distributions to model how attention and working memory capacity were impacted by the length of the retention interval.

\section{Experiment 1}

In Experiment 1, we characterized trial-by-trial fluctuations in the number of items maintained over time, while distinguishing between effects on the maximum number of items that could be stored, and an attention parameter that quantifies the probability that participants would achieve that maximum. Participants performed a discrete whole-report task which probed memory for each item on every trial, following either short $(1.5 \mathrm{~s})$ or long (10 s) retention intervals. We had participants maintain information over short (1.5 s) and long retention intervals (10 s) in order to determine whether longer retention intervals lead to declines in maximum working memory capacity (Kmax), or the probability of achieving $\operatorname{Kmax}(a)$, which we will refer to as attentional control. Previous research has numerically solved for Kmax and $a$ by running simulations (Adam et al., 2015). Here, we analytically solved for these two parameters. In addition, we examined whether failures to achieve maximum capacity were best characterized by a complete drop of all information from working memory, or a partial drop of some items while maintaining other items.

\section{Methods}

Participants. Twenty adults participated in Experiment 1 (8 male, 19-31 years, mean 23.25 years). Two additional participants were excluded for failing to complete the experiment in the allotted time ( 2 hours), resulting in an insufficient number of trials per condition $(<180)$. The final sample size for both experiments was selected a priori based on previous discrete whole-report sample sizes (Adam, Vogel, \& Awh, 2017). All participants in both experiments received payment 
(US \$20, \$10/hour) or course credit ( 2 credits, 1 credit/hour). Additionally, all participants reported normal or corrected-to-normal visual acuity and color vision and provided informed consent to a protocol approved by the University of Chicago Institutional Review Board.

Stimuli. Participants encoded arrays of six differently colored squares (subtending approximately $1^{\circ} \times 1^{\circ}$ ) on a gray background. Each square was one of nine possible colors without replacement (red, green, blue, yellow, magenta, cyan, white, black, orange). For the response screen, multicolored squares appeared that were a $3 \times 3$ grid of all nine colors. Participants were instructed to fixate on a black central dot $\left(0.43^{\circ}\right)$.

Apparatus. Stimuli were generated in MATLAB (The MathWorks, Natick, MA) using the Psychophysics toolbox (Brainard, 1997). Participants were seated approximately $67 \mathrm{~cm}$ from a 24inch LCD BenQ monitor. Squares could appear within approximately a $20^{\circ} \times 20^{\circ}$ area on the screen. To monitor compliance with the articulatory suppression instructions, audio was recorded through MATLAB with an Eberry microphone. 


\section{Discrete whole report working memory task}

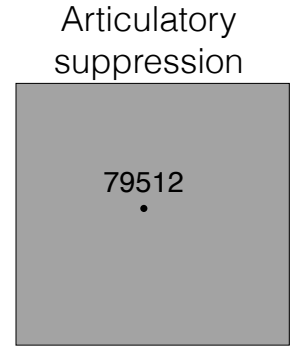

Until spacebar

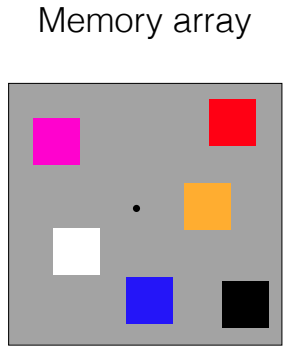

$150 \mathrm{~ms}$

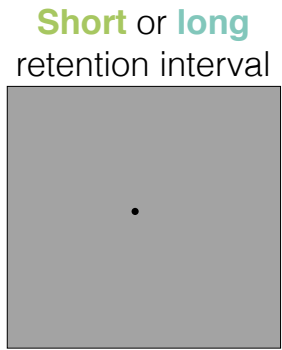

$1.5 \mathrm{~s}$ or $10 \mathrm{~s}$

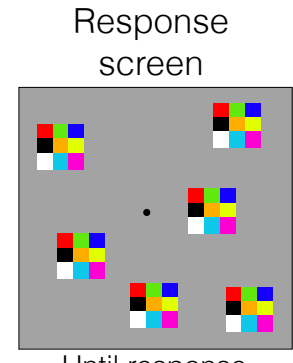

Until response

\section{Computational models}

\section{Maximum capacity plus guessing}

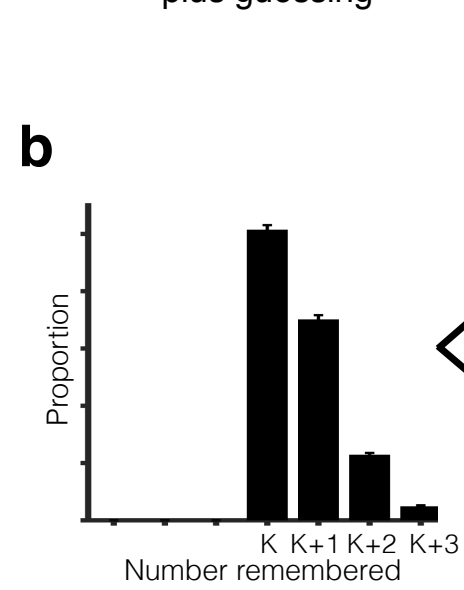

Attention

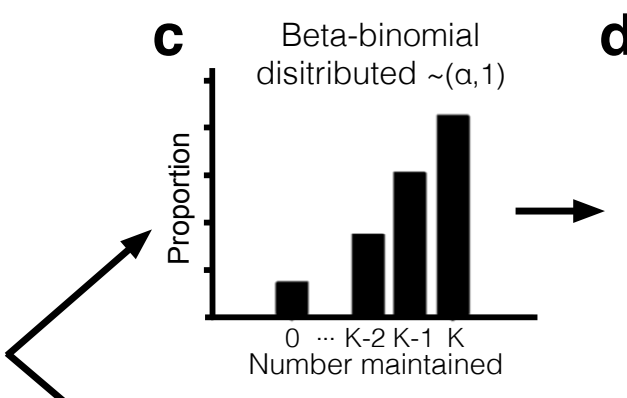

Predicted performance

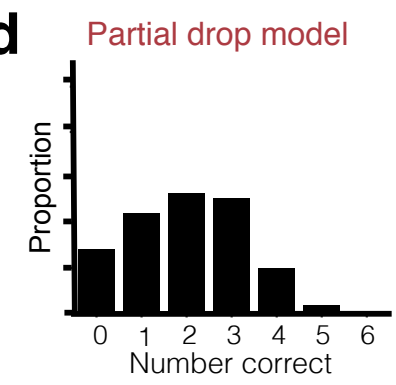

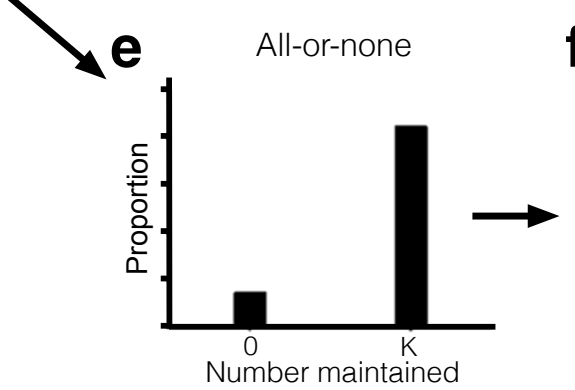

f

Complete drop model

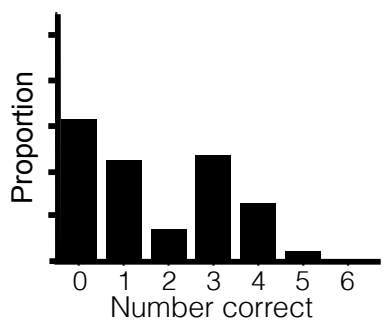

Figure 1 | Task design and computational model. a Discrete whole-report working memory task for Experiment 1. At the start of each trial, five digits appeared on the screen. Participants said these numbers at least once before trial initiation and repeated them continuously until the response screen appeared. Participants initiated each trial by pressing spacebar, and then a memory array of six colored squares appeared briefly $(150 \mathrm{~ms})$. The critical manipulation was the length of the retention interval, either short $(1.5 \mathrm{~s})$ or long $(10 \mathrm{~s})$. At the end of the retention interval, a response screen appeared with multicolored squares at the location of each original square. Participants selected the color of each square using the mouse. Participants were required to respond to all six items before the trial would proceed, and the multicolored squares remained on the screen until the participant finished responding. b Both models assume that participants have a fixed maximal capacity, plus guessing. $\mathbf{c}$ In the partial drop model, attentional control is distributed according to a beta-binomial function. $\mathbf{d}$ In the partial drop model, performance reflects a unimodal distribution. This model is essentially a partial loss of information, as participants can drop individual items, as opposed to the whole array. e In the complete drop model, attentional control is distributed according to a Bernoulli function. Attention to the array is therefore essentially all-or-none, as participants are either fully engaged and remember Kmax items, or they are fully disengaged and do not remember any of the items. f In the complete drop model, performance typically reflects a bimodal distribution. The distribution becomes unimodal if participants have extremely low working memory capacities. 
Procedure. Participants completed a discrete whole-report visual working memory task with articulatory suppression (Figure 1a). Prior to the onset of the memory array, five randomly generated digits appeared above the fixation dot. Participants said the numbers out loud at least once before pressing spacebar to begin the trial and continued to repeat the numbers until the response screen appeared. Participants encoded memory arrays of six colored squares that appeared briefly $(150 \mathrm{~ms})$, and then maintained these squares over a blank retention interval. Critically, the retention interval was either short (1.5 s, $50 \%$ of trials) or long (10 s, $50 \%$ of trials). After the retention interval, a response screen appeared with multicolored squares at the location of each original square. To respond, participants selected the color of the original square at each location using the mouse. Participants were required to respond to all 6 items before the trial would proceed, and all squares remained on the screen until the participant finished responding. Participants completed a block of 60 trials and then took a $30 \mathrm{~s}$ break. Within each block, the retention interval length was held constant (short or long). In total, participants completed 360 trials.

Analysis. Working memory performance was quantified per trial as the number of items for which the correct color was selected, ranging from 0 (no items correct) to 6 (all items correct). Across trials, we calculated the mean number of correct responses.

Computational models. In order to describe performance, we applied a family of computational models that have two parameters, one for attentional control $(a)$ and one for maximum working memory capacity (Kmax) (Figure 1b-f). These models assume that performance can be described using (1) a fixed maximal capacity (2) attentional control and (3) strategic guessing. 
In these models, each participant has a fixed maximal capacity. This capacity can vary from one item to the maximum number of items in the display $(\operatorname{Kmax} \in[1,6])$. On any given trial $(i)$, participants maintained some integer number of items $\left(N_{i}\right)$ in memory, ranging from 0 to their maximal capacity $\left(N_{i} \in[0, K \max ]\right)$.

We further assumed participants would strategically guess for the remaining items not maintained in memory. That is, for a given trial $(i)$, participants maintained an integer number of items with the correct color in mind $\left(N_{i}\right)$. For the remaining items $\left(6-N_{i}\right)$, we used a binomial distribution to model strategic guessing from among the remaining colors $\left(9-N_{i}\right)$.

These models also include a term for attentional control throughout the delay. Attentional control was operationalized as the probability of maintaining the maximum number of items (Kmax) for each trial. We examined two conceptualizations of attentional control in the different models:

In the complete drop model, attentional control was effectively binary: participants either maintained their maximum number of items $\left(N_{i}=K \max \right)$, or they had no information at all $\left(N_{i}=0\right)$. This model effectively reduces to a Bernoulli distribution over values zero and Kmax with the probability of Kmax being one minus the probability of potential failure.

In the partial drop model, the number of maintained items ranges over the integer numbers from 0 to Kmax. In this situation in statistics, we would typically apply the binomial distribution, which gives the probability distribution of zero to Kmax successes given a constant probability of success on each trial. However, we believe that the probability of success throughout an experiment is not constant. Prior evidence has shown that attentional control fluctuates over the course of an experiment and these attention fluctuations lead to fluctuations in working memory performance (deBettencourt et al., 2019). Therefore, we chose to use the beta-binomial distribution 
because this allowed the probability of success to also follow a probability distribution, rather than remain constant on each trial. For $n$ trials, this beta-binomial distribution is characterized by a beta function, $B(\alpha, \beta)$, and a binomial coefficient $\left(\frac{n}{K \max }\right)$, with the following probability density function:

$$
P(K \max \mid n, \alpha, \beta)=\frac{B(K \max +\alpha, n-K \max +\beta)\left(\frac{n}{K \max }\right)}{B(\alpha, \beta)}
$$

Model fitting. We computed the probability distributions for the complete and partial drop models over the 2D grid space of the free parameters for each model: $K \max \in[1,6]$ (with a step size of 1 ) and $a \in[0,10]$ (with a step size of 0.01 ). The complete drop model had one free parameter, $p$, which represented the probability of disengagement. The partial drop model also had one free parameter, $\alpha$, which represents the robustness of attentional control. Alpha determines the mean of the beta distribution for $p$ by mean $=\frac{\alpha}{\alpha+\beta}$. For this model, we kept the $\beta$ parameter equal to 1 , so that the beta distribution was $[\alpha, 1]$ for each trial. We were interested in the mean of the distribution, so we fixed $\beta$ equal to 1 and kept $\alpha$ free. By fixing $\beta$ to 1 , we assume that attentional control fluctuates quite a bit, especially when the success probability is intermediate. We then determined the best-fitting model to each individual participant's performance distributions by calculating which combination of parameters produced the largest log likelihood $(L L)$. To compare the complete and partial drop models, we used the Bayesian Information Criterion (BIC). We calculated the difference in BIC values between the two models. In this calculation, the penalty term of the BIC drops out because both models have the same number of free parameters (i.e. 1). We used the following formula to calculate the difference in BIC values between the two models:

$$
\Delta B I C=-2 \times\left(L L_{\text {complete }}-L L_{\text {graded }}\right)
$$


$L L_{\text {complete }}$ is the log-likelihood of the complete drop model, and $L L_{\text {graded }}$ is the log-likelihood of the partial drop model. Positive values of $\triangle B I C$ indicate a better fit for the partial than the complete drop model.

Statistics. Results are reported as the mean plus or minus the standard deviation, unless noted otherwise. Statistics in all experiments were computed using Student's two-tailed paired $t$ tests. Effect sizes are reported as Cohen's $d$.

\section{Results}

Behavioral performance. Working memory performance (Figure 2) was operationalized as the number of items correct per trial. Average working memory performance $(n)$ was calculated for each individual across all trials. If participants had been randomly guessing, chance performance would equal the probability of getting any item correct ( 1 of 9) multiplied by the number of items $(6$, chance $=0.67)$. Working memory performance across both conditions was well above chance $(n=2.40 \pm 0.85 ; t(19)=10.00, p<0.001)$.

Mean performance

a

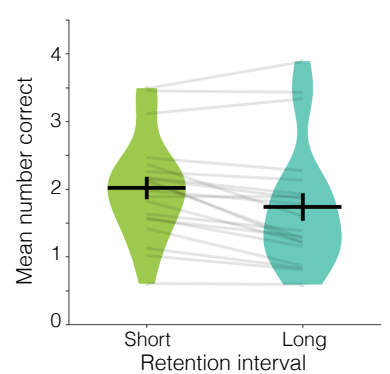

b

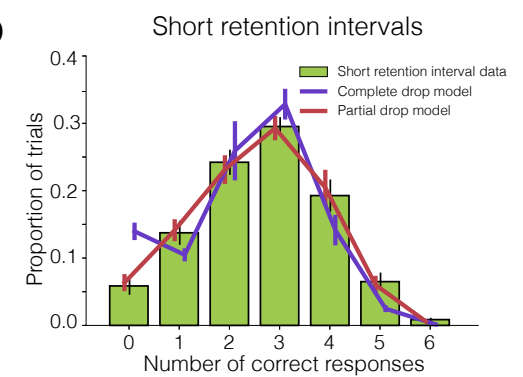

Distribution of performance \& model results

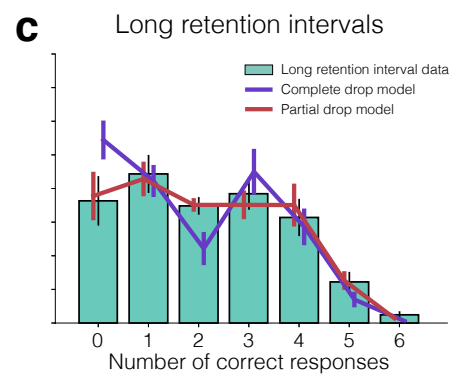

Figure 2 | Behavioral results and computational modeling fits for Experiment 1. a Working memory performance for short (green) vs. long (blue) retention intervals. Each dot represents the mean number correct in one condition for one participant. A line connects data from the same participant. The horizontal black line depicts the mean across participants. The black error bars reflect the standard error of the mean. The shaded area reflects the density across participants. b The green bars of the histogram depict the data from short retention interval trials. The height of each bar is the proportion of trials for each number of correct responses. Black error bars are the standard error of the mean across participants. The lines reflect the model fits using the best fitting parameters: complete drop model (purple) and partial drop model (red). Each model was fit independently for each participant, and error bars reflect the standard error of the mean of the best fitting parameters across participants $\mathbf{b}$ The data (blue bars) and best fitting models (purple and red lines) from long retention interval trials. 
The critical question was how performance differed over time (long vs. short retention intervals; Figure 2a). In line with prior work, we hypothesized that performance would decline following long retention intervals (Zhang \& Luck, 2009). We calculated the average number of correctly remembered items separately for trials with long and short retention intervals $\left(n_{\text {long }}=2.15 \pm 1.00, n_{\text {short }}=2.66 \pm 0.59\right)$. Indeed, participants remembered significantly fewer items after long vs. short retention intervals $(t(19)=4.35, p<0.001$, Cohen's $d=0.62)$.

Computational modeling. We developed an analytical solution to two computational models that could describe performance distributions: complete and partial drop models. These models make distinct predictions about the distribution of the number of correct responses across trials. The complete drop model was "all-or-none," such that participants either performed at their maximum capacity and remembered Kmax items, or they had no information about any of the items in the array, and, thus, maintained zero items. The partial drop model, on the other hand, assumed graded performance, such that participants could maintain anywhere from zero to Kmax items in working memory on any given trial. When $\operatorname{Kmax}$ is equal to 0 or 1 , these models make similar predictions.

First, we determined whether the complete or partial drop model better explained our data. To do this, we compared the fits between the models using a goodness-of-fit measure, BIC. Positive $B I C$ values indicate that the data are better fit by the partial drop model, whereas negative values indicate better fit by the complete drop model. In this experiment, both models made reasonable fits to data (Figure $\mathbf{2 b - c}$ ). However, data from both short and long retention intervals (Figure 2b-c) were better fit by the partial drop model $\left(B I C_{\text {long }}=1042.86, B C_{\text {short }}=1298.73\right)$ than the complete drop model. This suggests that performance fluctuates along a continuum and these fluctuations affect the maintenance of individual items in an array. 
Finally, the central question of interest was whether poorer performance following long retention intervals was best explained by a reduction in maximum working memory capacity or a reduction in the probability of achieving that maximum. The partial drop model showed that attentional control was reliably lower for long relative to short retention intervals $\left(a_{\text {long }}=1.28 \pm 2.20\right.$, $a_{\text {short }}=2.44 \pm 2.84 ; t(19)=3.30, p=0.004$; Cohen's $d=0.74$ ). However, there was not a reliable difference in maximum capacity (Kmax $\operatorname{mong}_{l}=3.55 \pm 0.55, \quad \operatorname{Kmax}_{\text {shorl }}=3.20 \pm 0.36 ; t(19)=-1.92$, $p=0.07$; Cohen's $d=-0.43)$. These results suggest that the performance decrements after long retention intervals reflect an inability to maintain attentional control throughout the longer retention intervals, not a reduction in the maximum number of items that could be stored in working memory.

\section{Discussion}

We developed a task that manipulated the length of the retention interval in a discrete whole-report working memory paradigm. Average performance declined after long vs. short retention intervals. We developed two models: the complete and the partial drop models, that both describe the number of items remembered as a result of some combination of attentional control and maximum working memory capacity. We found that the partial drop model better accounted for performance, which suggests that the amount of information in working memory across trials varied continuously. Additionally, the attentional control parameter reliably differed across retention intervals, such that it was lower after long retention intervals. However, the maximum working memory capacity parameter was equivalent for long and short retention intervals. Thus, decrements in performance after long retention intervals are due to a graded decline in sustaining attentional control, which leads to loss of individual items from working memory. 


\section{Experiment 2}

In Experiment 2, we sought to replicate Experiment 1 and extend our understanding of the role of attentional control during working memory maintenance. We interpreted the findings from Experiment 1 as reflecting variations in sustained attentional control during retention of information in working memory. In Experiment 1, however, we manipulated retention interval length across blocks, so, the differences that we found in Experiment 1 could be due to differences in preparation. For example, if participants know that they are going to have to retain information over a very long retention interval, they could better prepare than if they did not know the duration of the upcoming retention interval. To further eliminate potential differences in preparation across blocks, we intermixed trials of short and long retention intervals within blocks.

\section{Methods}

Participants. Twenty adults participated in Experiment 2 (10 male, 18-33 years, mean 24.05 years). Four additional participants were eliminated for technical issues with the experimental display code and four additional participants were excluded from analyses for leaving the experiment early.

Stimuli and apparatus. Same as Experiment 1.

Procedure. Same as Experiment 1, except short and long retention intervals were randomly inter-mixed across trials within each block. Participants completed a block of 48 trials and then took a $30 \mathrm{~s}$ break. In total, participants completed 288 trials.

Analysis, computational modeling, and statistics. Same as Experiment 1.

\section{Results}

Behavioral performance. Just as in Experiment 1, working memory performance (Figure 3) was operationalized as the number of items correct per trial, and average performance $(n)$ was 
calculated for each individual across all trials. Working memory performance on the discrete whole-report task was significantly above chance $(n=1.88 \pm 0.83 ; t(19)=6.61, p<0.001)$ suggesting that participants were not randomly guessing.

Mean performance

a

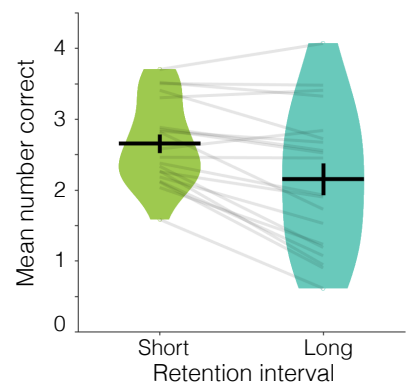

b

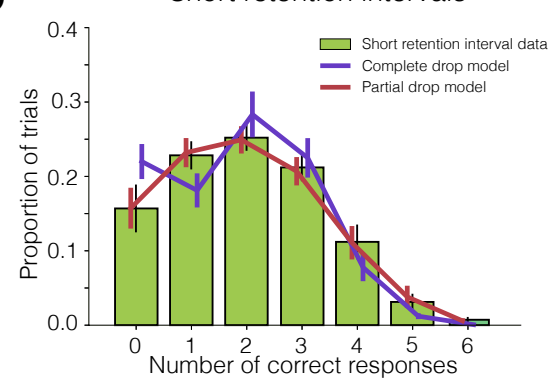

Distribution of performance \& model results

C

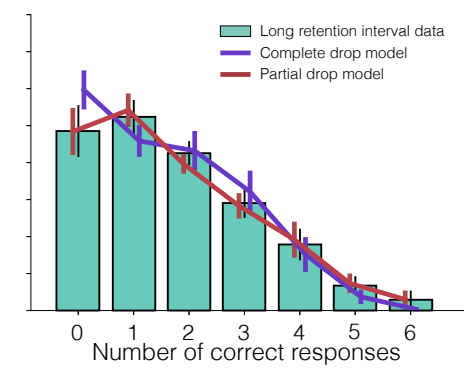

Figure 3 | Behavioral results and computational modeling fits for Experiment 2. a Working memory performance for short (green) vs. long (blue) retention intervals. Each dot represents the mean number correct in one condition for one participant. A line connects data from the same participant. The horizontal black line depicts the mean across participants. The black error bars reflect the standard error of the mean. The shaded area reflects the density across participants. $\mathbf{b}$ The green bars of the histogram depict the data from short retention interval trials. The height of each bar is the proportion of trials for each number of correct responses. Black error bars are the standard error of the mean across participants. The lines reflect the model fits using the best fitting parameters: complete drop model (purple) and partial drop model (red). Each model was fit independently for each participant, and error bars reflect the standard error of the mean of the best fitting parameters across participants b Data (blue bars) and best fitting models (purple and red lines) from long retention interval trials.

The critical question was whether working memory performance would again decline when

the retention intervals were longer and whether this decline was due to changes in the attentional control parameter or due to changes in maximum capacity (Figure 3a). We again observed worse performance after long retention intervals $\left(n_{\text {long }}=1.74 \pm 0.91, n_{\text {short }}=2.02 \pm 0.75 ; t(19)=3.73, p<0.001\right.$, Cohen's $d=0.34)$.

Computational modeling. We first compared our two computational models to determine whether the complete or partial drop model better explained our data. Once again, we found that both models made reasonable fits to the data (Figure 2b-c). However, just as in Experiment 1, we found that data from both the short and long retention intervals were better fit by the partial drop 
model $\left(B I C_{\text {long }}=590.38, B I C_{\text {shor }}=542.92\right)$ than the complete drop model. This finding reaffirms that performance across trials varies along a continuum and participants drop certain items from working memory while maintaining others over time.

Next, we investigated the parameter fits for attentional control and maximum working memory capacity for the partial drop model. We again found that attentional control was reliably lower during long than short retention intervals $\left(a_{\text {long }}=0.73 \pm 0.56, a_{\text {short }}=1.15 \pm 0.47 ; t(19)=4.59\right.$, $p<0.001$, Cohen's $d=1.03)$. Additionally, there was not a reliable difference across retention intervals between maximum capacity $\left(\right.$ Kmax $_{\text {long }}=3.00 \pm 1.30, \operatorname{Kmax}_{\text {short }}=2.95 \pm 0.65 ; t(19)=-0.24$, $p=0.82$; Cohen's $d=-0.05)$.

\section{Discussion}

In Experiment 2, we eliminated potential differences in preparation by randomly intermixing trials and, therefore, preventing participants from preparing for a certain length retention interval ahead of time. We replicated all of our findings from Experiment 1. Performance declined after long (vs. short) retention intervals. This decline in performance was graded, such that the amount of information in working memory across trials varied continuously. The maximum amount of information that could be maintained in working memory remained the same over both long and short retention intervals. However, the probability of maintaining this maximum amount of information in working memory declined for long compared to short retention intervals.

\section{General Discussion}

Past work has shown that variability in attention and filtering at encoding is an important limiting factor for working memory performance (Adam et al., 2015; Fukuda \& Vogel, 2011; Vogel, McCollough, \& Machizawa, 2005). That said, the decline in working memory performance 
with longer retention intervals - for which encoding demands are perfectly matched demonstrates that information is also lost even after successful encoding into working memory. Here, our main goals were to (1) characterize how the distributions of the number of items remembered varied across long and short retention intervals, and (2) use these distributions to characterize how the length of the retention interval impacts forgetting over time. Firstly, over two experiments, we observed that performance on a discrete whole-report task declined as retention interval increased (1.5 versus 10 seconds). Secondly, we found evidence in favor of the partial drop model, in which information loss over time is best described as a probabilistic loss of individual items rather than a complete failure of storage during specific trials. We developed a refined computational model of working memory performance that provides an analytical solution for estimating an attentional control parameter $(a)$ and a maximum capacity parameter (Kmax). We observed that when participants had to maintain information for a longer amount of time, the maximum amount of information (Kmax) that they could store in working memory remained the same. However, the probability of sustaining attentional control $(a)$ was lower after long compared to short retention intervals.

We found that performance decrements after long retention intervals were not driven by reductions in maximum capacity. However, precision of representations in working memory could still change over time, as has been previously suggested (Fougnie, 2008; Rademaker et al., 2018; Schneegans \& Bays, 2018). Our task provides a coarse measure of individual working memory representations and, thus, we cannot draw strong conclusions about changes in precision. However, even if precision does change over time, it may not lead to an aggregate increase in forgetting rates at the level of individual items. Future work could investigate how working 
memory precision changes over time by using a whole report task where participants report the precise color of every representation on every trial (e.g., Adam et al., 2017).

In sum, with this series of experiments, we extend our understanding of the impact of retention interval length on working memory performance. Past work has tended to portray capacity limits in working memory as reflecting a "ceiling" on storage capacity, similar to the limited space inside a container. From that perspective, it is plausible that factors that impair working memory performance yield a reduction in the available space in working memory. By contrast, we highlight recent work that suggests that variations in working memory performance across individuals may be better understood in terms of the probability of achieving one's maximum storage potential rather than in terms of differences in that potential (Adam et al., 2015). Likewise, we find that longer retention intervals reduce the probability of achieving one's maximum storage capacity, instead of reducing that storage capacity. Thus, longer retention intervals do not reduce the maximum amount of information that can be stored in working memory, but they provide more opportunity to forget individual items through lapses of attentional control. 


\section{Bibliography}

Adam, K. C. S., Mance, I., Fukuda, K., \& Vogel, E. K. (2015). The contribution of attentional lapses to individual differences in visual working memory capacity. Journal of Cognitive Neuroscience. https://doi.org/10.1162/jocn

Adam, K. C. S., Vogel, E. K., \& Awh, E. (2017). Clear evidence for item limits in visual working memory. Cognitive Psychology, 97, 79-97. https://doi.org/10.1016/j.cogpsych.2017.07.001

Awh, E., Vogel, E. K., \& Oh, S. H. (2006). Interactions between attention and working memory. Neuroscience, 139(1), 201-208. https://doi.org/10.1016/j.neuroscience.2005.08.023

Brainard, D. H. (1997). The Psychophysics Toolbox. Spatial Vision, 10(4), 433-436. https://doi.org/10.1163/156856897X00357

De Fockert, J. W., Rees, G., Frith, C. D., \& Lavie1, 3nilli. (2001). The Role of Working Memory in Visual Selective Attention. Science, 291(January), 1803-1806.

https://doi.org/10.1126/science.1056496

deBettencourt, M. T., Keene, P. A., Awh, E., \& Vogel, E. K. (2019). Real-time triggering reveals concurrent lapses of attention and working memory. Nature Human Behaviour. https://doi.org/10.1038/s41562-019-0606-6

Donkin, C., Nosofsky, R., Gold, J., \& Shiffrin, R. (2015). Verbal labeling, gradual decay, and sudden death in visual short-term memory. Psychonomic Bulletin \& Review, 22(1), 170178. https://doi.org/10.3758/s13423-014-0675-5

Fougnie, D. (2008). The relationship between attention and working memory. New Research on Short-Term Memory. https://doi.org/10.3389/conf.fnhum.2011.207.00576

Fukuda, K., \& Vogel, E. K. (2011). Individual differences in recovery time from attentional capture. Psychological Science, 22(3), 361-368.

https://doi.org/10.1177/0956797611398493

Lewandowsky, S., \& Oberauer, K. (2009). No evidence for temporal decay in working memory. Journal Of Experimental Psychology-Learning Memory And Cognition, 35(6), 1545-1551. https://doi.org/10.1037/a0017010

Oberauer, K., \& Lewandowsky, S. (2008). No Title. Psychological Review, 115(3), 544.

Rademaker, R. L., Park, Y. E., Sack, A. T., \& Tong, F. (2018). Evidence of gradual loss of precision for simple features and complex objects in visual working memory. Journal of Experimental Psychology. Human Perception and Performance.

Schneegans, S., \& Bays, P. M. (2018). Drift in neural population activity causes working memory to deteriorate over time. The Journal of Neuroscience, 3440-17. https://doi.org/10.1523/JNEUROSCI.3440-17.2018

Vogel, E. K., McCollough, A. W., \& Machizawa, M. G. (2005). Neural measures reveal individual differences in controlling access to working memory. Nature, 438(7067), 500503. https://doi.org/10.1038/nature04171

Zhang, W., \& Luck, S. J. (2009). Sudden death and gradual decay in visual working memory. Psychological Science, 20(4), 423-428. https://doi.org/10.1111/j.1467-9280.2009.02322.x 